\title{
Introduction to the Special Issue on Human-interaction- aware Data Analytics for Cyber-physical Systems
}

Cyber-physical Systems (CPS) are the systematic combination of physical processes, information, and communication technology. Typical examples of CPS are civil infrastructure monitoring, industrial control, and avionic systems. Such systems are generally deployed as a network of interacting elements with a huge amount of data. In addition, with the rapid advance of sensing, computing, and storing technologies, the amount of data increases tremendously. This imposes fundamental challenges on the design and management of CPS in multiple aspects, such as energy efficiency, security, privacy, reliability, scalability, and flexibility. Tackling these challenges necessitates innovative techniques of data analytics for handling the massive data in CPS.

In the design of innovative data analysis techniques, human activities and interactions with CPS must be taken into account since humans can monitor and control physical processes when interacting with very large data sets collected via sensors. However, various important human factors, e.g., predictable and repeating behavior, have not yet been well understood and fully taken into consideration in the current generation CPS. This special issue describes significant research contributions in the domain of human-interaction-aware data analytics for CPS.

This special issue consists of eight high-quality research articles selected from 18 submissions that had covered a broad range of topics. The article "Catering to Your Concerns: Automatic Generation of Personalized Security-centric Descriptions for Android Apps" develops an innovative scheme to help users avoid malware and privacy-breaching apps by generating security descriptions that explain the privacy- and security-related aspects of an Android app in clear and understandable terms. Taking human activities and interactions with cyberphysical systems into consideration, the article "Improved LDA Dimension Reduction-based Behavior Learning with Commodity WiFi for Cyber-physical Systems" designs a human-behaviorlearning system based on Channel State Information (CSI) utilizing a series of algorithms for data analysis and processing. To enable a sustainable edge computing paradigm while meeting the needs of home energy management and residents, the article "A Sustainable and User-behavior-aware Cyber-physical System for Home Energy Management” introduces a cyberphysical system based energy management framework. A novel system for drone surveillance is presented in the article entitled "A Crowdsensing-based Cyber-physical System for Drone Surveillance Using Random Finite Set Theory"; the proposed system utilizes surveillance data captured and sent from citizens' mobile devices to facilitate jointly drone detection and tracking. The article entitled "Energy-efficient ECG Signal Compression for User Data Input in Cyber-physical Systems by Leveraging Empirical Mode Decomposition" develops the first scheme of leveraging empirical mode decomposition (EMD) on ECG signals for sparse feature modeling and compression and further proposes a new ECG signal compression framework based on an EMD-constructed feature dictionary. The article "Socially Aware Path Planning for a Flying Robot in Close Proximity of Humans" describes a motion-planning framework in a cyber-physical system that takes into account the human's safety perception in the presence

(C) 2019 Copyright held by the owner/author(s).

2378-962X/2019/09-ART35e

https://doi.org/10.1145/3344260 
of a flying robot. The article entitled "A Distributed Tensor-train Decomposition Method for Cyber-physical-social Services" focuses on human-interaction-aware big data (HIBD) and presents a distributed Tensor-train (DTT) decomposition method to process the high-order and largescale HIBD. Finally, to adapt to the changes in a driver's controllability, the human-interactionaware adaptive functional safety processing for multi-functional automotive cyber-physical systems in two main phases is studied in the article entitled "Human-interaction-aware Adaptive Functional Safety Processing for Multi-functional Automotive Cyber-physical Systems."

Last but not least, we express our sincere thanks to all authors who submitted their articles to this special issue. We are also indebted to the reviewers for their valuable time and great efforts in enhancing the quality of the accepted articles. A special appreciation is extended to Professor Tei-Wei Kuo, the Editor-in-Chief of ACM Transactions on Cyber-Physical Systems, and his editorial team for their consistent guidance and assistance in the preparation and publication of this special issue.

Tongquan Wei East China Normal University

Junlong Zhou Nanjing University of Science and Technology

Rajiv Ranjan Newcastle University

Isaac Triguero University of Nottingham

Huafeng Yu Boeing Research \& Technology

Chun Jason Xue City University of Hong Kong

Schahram Dustdar TU Wien

Guest Editors 